\title{
Stroke incidence and case fatality: a 9-year prospective population-based study in an elderly population of Bagheria, Italy
}

\author{
Valentina Arnao ${ }^{1,2}$ (D) Giuseppe Salemi ${ }^{1}$ (D) $\cdot$ Salvatore Scondotto ${ }^{3} \cdot$ Nicola Casuccio $^{4} \cdot$ Marianna Riolo $^{5}$ (D) \\ Marco D'Amelio ${ }^{1}$ (D) P Paolo Ragonese ${ }^{1}$ (D) P Paolo Aridon ${ }^{1}$ (I)
}

Received: 26 April 2020 / Accepted: 13 October 2020 / Published online: 19 October 2020

(C) The Author(s) 2020

\begin{abstract}
Background The incidence of stroke in high-income countries has been on the decline; however, few epidemiological surveys have been conducted in recent years to specifically estimate the incidence along with outcome of stroke, in Italy. This study aimed to examine the incidence and case fatality rates of stroke in an elderly Italian population.

Methods A cohort of 2200 people $>65$ years was randomly stratified from the total elderly population of Bagheria, Italy. A 9year prospective population-based study was performed (19,800 person/years).

Results We identified 112 first-ever strokes, 53 females and 59 males: 82 (73.1\%) ischemic, 13(11.6\%) intracerebral haemorrhages, $6(5.35 \%)$ subarachnoid haemorrhages, while $11(9.8 \%)$ were classified as undetermined strokes. The crude overall annual incidence was 5.65 per 1000 (95\%CI: 4.61 to 6.70) for first-ever stroke. The overall crude incidence rates were 4.74 per 1000 (5.08 for males and 4.46 for females) for ischemic stroke, 0.65 ( 0.99 for males and 0.37 for females) for intracerebral haemorrhage, and 0.03 for subarachnoid haemorrhage. The incidence rate for first-ever stroke was 5.4 per 1000 (95\% CI: 5.36 to 5.45) after adjustment for the 2015 World population and 5.56 (95\% CI: 5.52 to 5.61 ), compared to the 2015 European population. Overall case fatality rates for first-ever stroke was $8.19 \%$ at 28 days and $24.1 \%$ at 1 year.

Conclusion Our study shows that in the elderly population investigated, stroke incidence and case fatality rates resulted being lower, compared to those from Italian and most European populations. Similar to previous studies, these rates increased linearly with age and were higher in males.
\end{abstract}

Keywords Epidemiology $\cdot$ Prevalence $\cdot$ Incidence $\cdot 28$-day case fatality rate $\cdot$ Stroke

Paolo Aridon

paolo.aridon@unipa.it

1 Department of Biomedicine, Neuroscience and Advanced Diagnostics (BiND), University of Palermo, Via Gaetano la Loggia n.1, 90129 Palermo, Italy

2 UO Neurologia e Stroke Unit, A. R. N. A. S. Ospedali Civico Di Cristina Benfratelli, Palermo, Italy

3 Sorveglianza Ed Epidemiologia Valutativa-Regione Sicilia, Palermo, Italy

4 Azienda sanitaria Provinciale di Palermo -UOC di sanità pubblica, epidemiologia e medicina preventiva, Palermo, Italy

5 Ospedale Santa Croce di Moncalieri - Asl TO5, Moncalieri, Torino, Italy

\section{Introduction}

Stroke remains a major health problem worldwide. In highincome countries, it represents the second cause of death and the third leading cause of disability [1], whereas low- and middle-income countries have been experiencing an increased incidence over the last years [2].

Stroke is a complex disease resulting from the interaction of numerous environmental and genetic factors. A detailed investigation of lifestyle, diet, and genetic predisposition cannot be separated from epidemiological rates, especially incidence and mortality. In fact, changes in trends for both incidence and mortality rates are important in identifying the effects of changes in modifiable risk factors, screening factors, and prevention on stroke trends. Our objective was to assess for the incidence along with the 28-day and 1-year case 
Table 1 Characteristics of patients with first-ever stroke in Bagheria, according to the type of index stroke

\begin{tabular}{|c|c|c|c|c|c|}
\hline & Total $(=112)$ & IS $(=82)$ & $\mathrm{ICH}(=13)$ & $\mathrm{SAH}(=6)$ & Unknown $(=11)$ \\
\hline Male $(\%)$ & $59(52.6)$ & $40(48.7)$ & $9(69.2)$ & $4(66.6)$ & $6(54.5)$ \\
\hline Age (SD) & $79.2( \pm 5.9)$ & $79.0( \pm 5.6)$ & $81.1( \pm 7.3)$ & $76.4( \pm 5.2)$ & $79.5( \pm 6.6)$ \\
\hline \multicolumn{6}{|l|}{ Education (\%) } \\
\hline Elementary or below & $92(82.1)$ & $66(80.4)$ & $11(84.6)$ & $5(83.3)$ & $10(90.9)$ \\
\hline Middle school & $11(9.8)$ & $9(10.9)$ & $1(7.6)$ & $1(16.7)$ & 0 \\
\hline High school or above & $9(8.1)$ & $7(8.5)$ & $1(7.6)$ & 0 & $1(9.1)$ \\
\hline \multicolumn{6}{|l|}{ Smoking status (\%) } \\
\hline Never & $53(47.3)$ & $45(54.8)$ & $4(30.8)$ & $1(16.7)$ & $3(27.2)$ \\
\hline Past smoker & $11(9.8)$ & $6(7.3)$ & $2(15.4)$ & $1(16.7)$ & $2(18.1)$ \\
\hline Current smoker & $41(36.6)$ & $27(32.9)$ & $5(38.5)$ & $4(66.7)$ & $5(45.4)$ \\
\hline Unknown & $7(6.3)$ & $4(4.8)$ & $2(15.4)$ & 0 & $1(9.1)$ \\
\hline \multicolumn{6}{|l|}{ Drinking status (\%) } \\
\hline Never & $62(55.4)$ & $50(60.9)$ & $5(38.5)$ & $2(33.3)$ & $5(45.4)$ \\
\hline Past drinker & $3(2.7)$ & $1(1.2)$ & $1(7.7)$ & 0 & $1(9.1)$ \\
\hline Current drinker & $40(35.7)$ & $27(32.9)$ & $5(38.5)$ & $4(66.7)$ & $4(36.3)$ \\
\hline Unknown & $7(6.3)$ & $4(4.8)$ & $2(15.4)$ & 0 & $1(9.1)$ \\
\hline \multicolumn{6}{|l|}{ BMI $\left(\mathrm{kg} / \mathrm{m}^{2}\right)$} \\
\hline$<18.5$ & 0 & 0 & 0 & 0 & 0 \\
\hline $18.6-24.9$ & $27(24.1)$ & $22(26.8)$ & $5(38.5)$ & 0 & 0 \\
\hline $25-29.9$ & $45(40.1)$ & $29(35.3)$ & $3(23.1)$ & $4(66.7)$ & $9(81.8)$ \\
\hline$\geq 30$ & $20(17.8)$ & $15(18.2)$ & $3(23.1)$ & $1(16.7)$ & $1(9.1)$ \\
\hline Unknown & $20(17.8)$ & $16(19.5)$ & $2(15.4)$ & $1(16.7)$ & $1(9.1)$ \\
\hline \multicolumn{6}{|l|}{ Hypertension (\%) } \\
\hline Yes & $65(58)$ & $47(57.3)$ & $7(53.8)$ & $4(66.7)$ & $7(63.6)$ \\
\hline No & $39(34.8)$ & $30(36.5)$ & $4(30.8)$ & $2(33.3)$ & $3(27.2)$ \\
\hline Unknown & $8(7.1)$ & $5(6.2)$ & $2(15.4)$ & 0 & $1(9.1)$ \\
\hline \multicolumn{6}{|l|}{ Diabetes $(\%)$} \\
\hline Yes & $27(24.1)$ & $22(26.8)$ & $2(15.4)$ & $1(16.7)$ & $2(18.1)$ \\
\hline No & $78(69.6)$ & $56(68.2)$ & $9(69.2)$ & $5(83.3)$ & $8(72.7)$ \\
\hline Unknown & $7(6.3)$ & $4(4.9)$ & $2(15.4)$ & 0 & $1(9.1)$ \\
\hline \multicolumn{6}{|l|}{ Dyslipidemia (\%) } \\
\hline Yes & $22(19.6)$ & $16(19.5)$ & $2(15.4)$ & 0 & $4(36.3)$ \\
\hline No & $83(74.1)$ & $62(75.6)$ & $9(69.2)$ & $6(100)$ & $6(54.5)$ \\
\hline Unknown & $7(6.2)$ & $4(4.8)$ & $2(15.4)$ & 0 & $1(9.1)$ \\
\hline \multicolumn{6}{|l|}{ TIA (\%) } \\
\hline Yes & $6(5.4)$ & $5(6.1)$ & $1(7.7)$ & 0 & 0 \\
\hline No & $91(81.3)$ & $63(76.8)$ & $12(92.3)$ & $6(100)$ & $10(90.9)$ \\
\hline Unknown & $15(13.4)$ & $14(17.0)$ & 0 & 0 & $1(9.1)$ \\
\hline \multicolumn{6}{|c|}{ Cardiovascular diseases (\%) } \\
\hline Yes & $36(32.1)$ & $23(28.0)$ & $5(38.5)$ & $2(33.3)$ & $6(54.5)$ \\
\hline No & $69(61.6)$ & $55(67.0)$ & $6(46.2)$ & $4(66.7)$ & $4(36.3)$ \\
\hline Unknown & $7(6.3)$ & $4(4.9)$ & $2(15.4)$ & 0 & $1(9.1)$ \\
\hline \multicolumn{6}{|c|}{ Medications before admission (\%) } \\
\hline Antihypertensive med. & $46(41.1)$ & $35(42.6)$ & $3(23.1)$ & $2(33.3)$ & $6(54.5)$ \\
\hline Antithrombotic med. & $3(2.7)$ & $3(3.2)$ & 0 & 0 & 0 \\
\hline Diabetes med. & $25(22.3)$ & $20(24.3)$ & $2(15.4)$ & $1(16.7)$ & $2(18.1)$ \\
\hline Lipid-lowing med. & $18(16.1)$ & $14(17.1)$ & $1(7.7)$ & 0 & $3(27.2)$ \\
\hline
\end{tabular}

$B M I$, body mass index; $I C H$, intracerebral haemorrhage; $I S$, ischemic stroke; $S A H$, subarachnoid haemorrhage; $\mathrm{Med}$, medicines. Unless indicated otherwise, data are given as $n(\%)$ or the mean $\pm \mathrm{SD}$ 
Table 2 Age- and sex-specific incidence rates of cerebrovascular diseases and first-ever stroke per 1000 person-years in Bagheria, an elderly community: Incidence of first-ever stroke

\begin{tabular}{|c|c|c|c|c|c|c|c|c|c|}
\hline \multirow[t]{2}{*}{ Age group } & \multicolumn{3}{|l|}{ Males } & \multicolumn{3}{|c|}{ Females } & \multicolumn{3}{|l|}{ All } \\
\hline & $\mathrm{P} / \mathrm{y}$ & Cases & Rate & $\mathrm{P} / \mathrm{y}$ & Cases & Rate & $\mathrm{P} / \mathrm{y}$ & Cases & Rate \\
\hline $65-69$ & 2043 & 9 & 4.40 & 2394 & 8 & 3.34 & 4437 & 17 & 3.83 \\
\hline $70-74$ & 2898 & 17 & 5.86 & 2925 & 16 & 5.47 & 5823 & 33 & 5.66 \\
\hline $75-79$ & 2142 & 16 & 7.47 & 2664 & 12 & 4.50 & 4806 & 28 & 5.82 \\
\hline $80-84$ & 1341 & 14 & 10.44 & 1611 & 11 & 6.82 & 2952 & 25 & 8.46 \\
\hline $85+$ & 621 & 3 & 4.83 & 1161 & 6 & 5.16 & 1782 & 9 & 5.05 \\
\hline \multirow[t]{4}{*}{ All ages } & 9045 & 59 & 6.52 & 10,755 & 53 & 4.92 & 19,800 & 112 & 5.65 \\
\hline & & & *6.09 & & & $* 4.67$ & & & $* 5.40$ \\
\hline & & & \#6.29 & & & $\# 4.79$ & & & \#5.56 \\
\hline & & & ${ }^{\circ} 6.34$ & & & ${ }^{\circ} 4.85$ & & & ${ }^{\circ} 5.62$ \\
\hline
\end{tabular}

*Adjusted to the world population; ${ }^{\#}$ Adjusted to the European population; ${ }^{\circ}$ Adjusted to the Italian population fatality rates of first-ever stroke in a Sicilian population using data from a population-based survey of elderly participants.

\section{Methods}

In a previous paper, we described a survey (Prevalence day September 30,2006) that was carried out in the municipality of Bagheria, a town nearby Palermo. Briefly, a cohort of 2200 people over 65 years of age, representing $25 \%$ of the whole population, was randomly stratified [3]. A semi-structured questionnaire was administered to each subject and, as a second level, a neurologist evaluated people reporting neurological symptoms. Nine years later, the clinical data for the whole cohort were obtained from a local health institution and from the general practitioners (GPs) of the study area. In addition, we examined the Hospital Discharge Diagnosis Data Bank, a computerized system that collects the diagnosis of each hospitalized patient of the study area. We primarily looked for patients having a discharge diagnosis including codes 430 to 438 , according to the International Classification of Disease, 9th revision. Mortality, cause of mortality data, and death certificates were obtained from local institutions. In particular, the local Health Registry of the Sanitary District (ASP 6) provided the dates along with information on dates and causes of death.

According to the WHO criteria, stroke was considered "rapidly developing clinical symptoms or signs of focal, and at times global, loss of cerebral function, with symptoms lasting more than 24 hours or leading to death, with no apparent cause other than that of vascular origin" [4]. In this follow-up study, we included only cases of first-ever stroke, considering it as an event occurring for the first time in a patient's lifetime. Discharge diagnosis codes, clinical data obtained from either hospitalization or GPs permitted us to categorize ischemic stroke (IS), intracerebral haemorrhage (ICH), subarachnoid
Fig. 1 Age- and sex-specific incidence rates of first-ever stroke in Bagheria

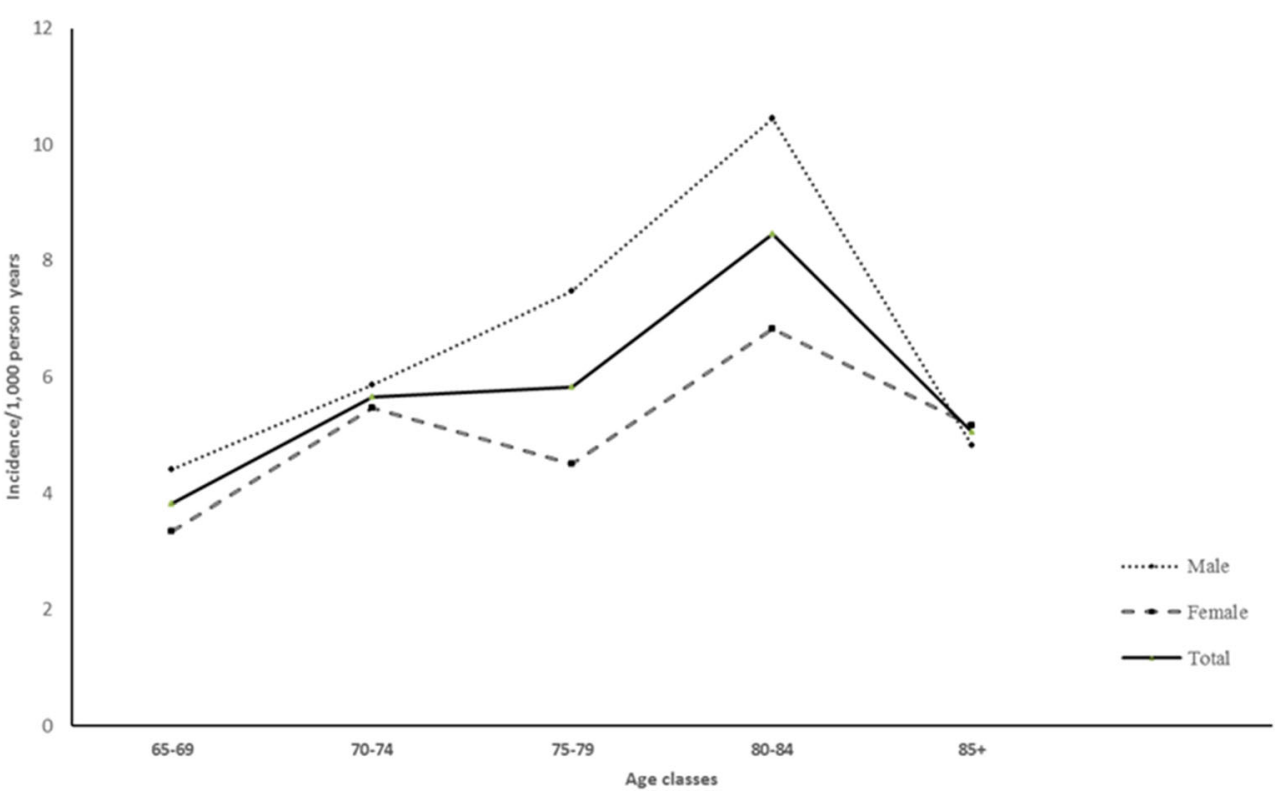


Table 3 Age- and sex-specific incidence rates of cerebrovascular diseases and first-ever stroke per 1000 person-years in Bagheria, an elderly community: Incidence of ischemic stroke

\begin{tabular}{|c|c|c|c|c|c|c|c|c|c|}
\hline \multirow[t]{2}{*}{ Age Group } & \multicolumn{3}{|c|}{ Males } & \multicolumn{3}{|c|}{ Females } & \multicolumn{3}{|l|}{ All } \\
\hline & $\mathrm{P} / \mathrm{y}$ & Cases & Rate & $\mathrm{P} / \mathrm{y}$ & Cases & Rate & $\mathrm{P} / \mathrm{y}$ & Cases & Rate \\
\hline $65-69$ & 2043 & 7 & 3.42 & 2394 & 7 & 2.92 & 4437 & 14 & 3.15 \\
\hline $70-74$ & 2898 & 12 & 4.14 & 2925 & 12 & 4.10 & 5823 & 24 & 4.127 \\
\hline $75-79$ & 2142 & 10 & 4.66 & 2664 & 10 & 3.75 & 4806 & 20 & 4.16 \\
\hline $80-84$ & 1341 & 9 & 6.71 & 1611 & 9 & 5.58 & 2952 & 18 & 6.09 \\
\hline $85+$ & 621 & 2 & 3.22 & 1161 & 4 & 3.44 & 1782 & 6 & 3.36 \\
\hline All ages & 9045 & 40 & 4.42 & 10,755 & 42 & 3.90 & 19,800 & 82 & 4.14 \\
\hline
\end{tabular}

haemorrhage (SAH), or undetermined stroke (UND). Patients with transient ischemic attacks (TIA) or recurrent stroke were not included in the analysis of "first-ever group".

The subjects were re-evaluated at the end of follow-up period (December 31, 2015). Crude-, age-, and sexspecific incidence rates with $95 \%$ confidence intervals were calculated. Person/years were obtained multiplying the mean of the population by nine (length of the followup period). All rates were adjusted to the 2015 World, European, and Italian population [5]. The 28-day and 1year case fatality rates were defined as the number of deaths due to stroke (or stroke type) divided by the total number of cases of stroke (or stroke type). The local Ethical and Scientific Committee (Palermo 1, v. 5/2015, May 13, 2015) approved this study.

\section{Results}

We identified 176 patients with cerebrovascular disease during the follow-up period. Eighty-four $(47.3 \%)$ were female with a mean age of 76 (range 65 to 92) and ninety-two $(52.7 \%)$ were male with a mean age of 75 (65 to 87$)$. Of these $176,112(63.6 \%)$ were first-ever strokes (patients' characteristics have been summarized in Table 1), 59 were male with a mean age of 75 years (range 65 to 87), and 53 were female (mean age 76 years); 53 (29.5\%) were transient ischemic attacks and 11 were described as code 438 (late effects of cerebrovascular disease). Almost all patients underwent neuroimaging study. Hence, one hundred and twelve cases were included in the study: 82 were classified as ischemic stroke (73.1\%), 13 intracerebral haemorrhages (11.6\%), and 6 subarachnoid haemorrhages $(5.35 \%)$; 11 were classified as undetermined strokes $(9.8 \%)$ because they did not undergo a neuroimaging test.

The overall crude incidence rate for first-ever stroke $(N=$ 112) was 5.65 per 1000 (95\%CI: 4.61 to 6.70$)$, showing higher rates in males 6.52 (95\% CI: 4.86 to 8.18), compared to females (4.92 per 1000; $95 \%$ CI: 3.60 to 6.25 ). Therefore, the incidence rate adjusted to the 2015 World population was 5.40 (95\%CI: 5.22 to 5.58 ): 6.09 (95\%CI: 5.79 to 6.38 ) for males and 4.67 (95\%CI: 4.43 to 4.90 ) for females.

The incidence rates adjusted to the European and Italian population were 5.56 (95\% CI: 5.16 to 5.96 ) and 5.62 (95\%CI: 4.35 to 6.89 ), respectively (Table 2 ).

Age-specific incidence rates of first-ever stroke increased with advancing age in both sexes, with a peak of 8.46 per 1000 in narrow 5-year age classes after the age of 80 years, 10.44 per 1000 for males and 6.82 per 1000 for females (Fig. 1 and Table 2).

Regarding the different subtypes of stroke, the overall crude incidence rates were 4.14 (4.42 for males and 3.90 for females) per 1000 for ischemic stroke, 0.65 (0.99 for males and 0.37 for females) for intracerebral haemorrhage and 0.30 ( 0.44 for males and 0.18 for females) for subarachnoid haemorrhage (Tables 3, 4, and 5). Once again, these values rose
Table 4 Age- and sex-specific incidence rates of cerebrovascular diseases and first-ever stroke per 1000 person-years in Bagheria, an elderly community: Incidence of intracerebral haemorrhage

\begin{tabular}{|c|c|c|c|c|c|c|c|c|c|}
\hline \multirow[t]{2}{*}{ Age group } & \multicolumn{3}{|c|}{ Males } & \multicolumn{3}{|c|}{ Females } & \multicolumn{3}{|l|}{ All } \\
\hline & $\mathrm{P} / \mathrm{y}$ & Cases & Rate & $\mathrm{P} / \mathrm{y}$ & Cases & Rate & $\mathrm{P} / \mathrm{y}$ & Cases & Rate \\
\hline $65-69$ & 2043 & 1 & 0.48 & 2394 & 0 & 0.0 & 4437 & 1 & 0.22 \\
\hline $70-74$ & 2898 & 2 & 0.69 & 2925 & 2 & 0.68 & 5823 & 4 & 0.68 \\
\hline 75-79 & 2142 & 4 & 1.86 & 2664 & 0 & 0.0 & 4806 & 4 & 0.83 \\
\hline $80-84$ & 1341 & 2 & 1.49 & 1611 & 0 & 0.0 & 2952 & 2 & 0.67 \\
\hline $85+$ & 621 & 0 & 0 & 1161 & 2 & 1.72 & 1782 & 2 & 1.12 \\
\hline All ages & 9045 & 9 & 0.99 & 10,755 & 4 & 0.37 & 19,800 & 13 & 0.65 \\
\hline
\end{tabular}


Table 5 Age- and sex-specific incidence rates of cerebrovascular diseases and first-ever stroke per 1000 person-years in Bagheria, an elderly community: Incidence of subarachnoid haemorrhage

\begin{tabular}{|c|c|c|c|c|c|c|c|c|c|}
\hline \multirow[t]{2}{*}{ Age group } & \multicolumn{3}{|l|}{ Males } & \multicolumn{3}{|c|}{ Females } & \multicolumn{3}{|l|}{ All } \\
\hline & $\mathrm{P} / \mathrm{y}$ & Cases & Rate & $\mathrm{P} / \mathrm{y}$ & Cases & Rate & $\mathrm{P} / \mathrm{y}$ & Cases & Rate \\
\hline $65-69$ & 2043 & 1 & 0.48 & 2394 & 1 & 0.41 & 4437 & 2 & 0.45 \\
\hline $70-74$ & 2898 & 2 & 0.69 & 2925 & 0 & 0 & 5823 & 2 & 0.34 \\
\hline $75-79$ & 2142 & 0 & 0 & 2664 & 1 & 0.37 & 4806 & 1 & 0.20 \\
\hline $80-84$ & 1341 & 1 & 0.74 & 1611 & 0 & 0.0 & 2952 & 1 & 0.33 \\
\hline $85+$ & 621 & 0 & 0 & 1161 & 0 & 0 & 1782 & 0 & 0 \\
\hline All ages & 9045 & 4 & 0.44 & 10,755 & 2 & 0.18 & 19,800 & 6 & 0.30 \\
\hline
\end{tabular}

with age and reached a peak between 80 and 84 years of age for ischemic, and 75-79 years and over 85 years, respectively, for males and females for intracerebral haemorrhage.

The overall mean age of death was 82.6 years $(81.3$ for males and 84.2 for females). The higher mortality (54\%) was for intracerebral haemorrhage ( $7 / 13$ patients) compared to subarachnoid haemorrhage $(50 \% ; 3 / 6$ patients) and ischemic stroke (44\%; $41 / 93$ patients). The overall case fatality rates at 28 and 30 days were equal at $8.9 \%$ (10/112 patients): $10.3 \%$ for males $(6 / 58$ patients, mean age of death 78 years) and $7.4 \%$ for females (4/54 patients, mean age of death 83 years). The overall 1-year case fatality rate was 24.1 (27/ 112 patients; mean age of death 81$): 27.5 \%$ for males $(16 / 58$ patients; mean age at death 80 years) and $20.3 \%$ for females (11/54 patients, mean age at death 82 years).

\section{Discussion}

Over the last years, several studies on the incidence and case fatality of stroke have been conducted, but it must be considered that a comparison of results cannot always be possible, due to differing study designs and types of population used. In this population-based study, we report on the incidence and case fatality rates for first-ever stroke, in an elderly population from a Sicilian municipality.

We report an incidence rate lower than those obtained by other studies using European populations. Similar to previous studies, incidence increased linearly with age and was higher in males than in females. It is worth noting that Manobianca et al. [6] reported, for the age groups 65-74, 75-84, and over 85 , incidence rates of $3.5,11$, and 21.4 per 1000 , respectively, while Musolino et al. [7] for the same age groups reported rates of $7.46,9.65$, and 12.18 , respectively. In our study, the lower incidence confirms recent scientific evidence of a decline in stroke incidence in Italy [8], reported by a 10-year population-based study carried out in the Lombardy Region in Northern Italy. Moreover, these decreased rates may also have been attributable to protective factors related to varied cultures and lifestyles in the different study areas. Thus, adherence to the Mediterranean diet, a better control of cardiovascular risk factors, especially smoking habits and alcohol consumption, could make our results unsurprising.

In our study, higher incidence rates were found for males. The lower frequency of smoking and alcohol consumption in older females, in addition to a lack of exposure to environmental factors (e.g. agricultural chemicals, herbicide), could partially explain these results.

The distribution of different stroke subtypes was similar to those reported in other studies, but our incidence was proportionally lower.

In previous European studies, 30-day case fatality ranged from $18.1 \%$ in Acquaviva Casamassima [9] to 20.3 in Trasimeno [10] or 23.7 in Vibo Valentia (28-day case fatality) [11]. Likewise, the 1-year case fatality varied from 39.1 on the Aeolian Island [12] to 40.2 in Vibo Valentia [11]. In our population, the 30-day and 1-year mortality rates were the lowest reported in Italy $[13,14]$. Despite this, it could be easy to hypothesise that these differences may be due to an improved global health; however, we must consider that this is the first study to be conducted on this area in 30 years and to compare the results from different geographical areas could lead to unreliable conclusions. Furthermore, we cannot consider our data as the result of an improvement in standard of care provided by stroke unit care, as the closest stroke units to the study area were at least $20 \mathrm{~km}$ away, and an efficient stroke network for expedient transfer is lacking. It must be stated here that none of the patients included in this study received thrombolysis and/or thrombectomy. Moreover, we can hypothesise that the observed better survival in the elderly population may be due to a real improvement in the quality of medical care for stroke patients at Sicilian hospitals, or to an underdiagnosis of severe stroke, as these patients probably never sought out hospital care or did not have the possibility to reach an emergency care area. In addition, general practitioners filling out death certificates could not include stroke as the cause of death.

Despite a 9-year-period of observation, the magnitude of the sample size could be considered a limitation. On the contrary, the present study has several strengths. First, it was 
conducted in a well-defined geographical district in Sicily, including all the multiple sources available to ascertain and characterize stroke occurrence. Second, the clinical definition, according to the WHO criteria, and the choice to include in the analyses only the first-ever strokes, considering the information on stroke subtypes, permitted for the comparison with other studies.

In conclusion, the findings of this study suggest that this Sicilian population, as the Southern Italian population and other industrialized European countries, needs to be monitored for incidence and case fatality due to cerebrovascular disease, with a particular attention paid toward identifying stroke subtype, mortality, and any risk factors that allow for the development of effective public health approaches aimed at preventing stroke.

Acknowledgements Open access funding provided by Università degli Studi di Palermo within the CRUI-CARE Agreement. The authors would like to acknowledge all patients and their relatives involved in the study. Data availability The data that support the findings of this study are available from the corresponding author upon reasonable request.

\section{Compliance with ethical standards}

Conflict of interest The authors declare that they have no conflict of interest.

Ethical approval The study protocol has been approved by the local Ethics Committee (Palermo 1). Written Informed consent was obtained from all individual participants included in the study.

Open Access This article is licensed under a Creative Commons Attribution 4.0 International License, which permits use, sharing, adaptation, distribution and reproduction in any medium or format, as long as you give appropriate credit to the original author(s) and the source, provide a link to the Creative Commons licence, and indicate if changes were made. The images or other third party material in this article are included in the article's Creative Commons licence, unless indicated otherwise in a credit line to the material. If material is not included in the article's Creative Commons licence and your intended use is not permitted by statutory regulation or exceeds the permitted use, you will need to obtain permission directly from the copyright holder. To view a copy of this licence, visit http://creativecommons.org/licenses/by/4.0/.

\section{References}

1. Feigin VL, Norrving B, Mensah GA (2017) Global burden of stroke. Circ Res 120(3):439-448

2. Owolabi M, Johnson W, Khan T, Feigin V, for Operations Committee of the Lancet Neurology on Stroke (2018) Effectively combating stroke in low- and middle-income countries: placing proof in pragmatism - the lancet neurology commission. Journal of Stroke Medicine 1(1):65-67

3. Ragonese P, Aridon P, D'Amelio M, Aiello F, Antonietta Mazzola M, Realmuto S, Salemi G, Savettieri G (2011) A population-based survey of cognitive performance in a Sicilian elderly community. Neuroepidemiology 36(3):169-176

4. Hatano S (1976) Experience from a multicentre stroke register: a preliminary report. Bull World Health Organ 54(5):541-553

5. Population division of the Department of Economic and social affairs of the United Nations Secretariat, desa / population division World Population Prospects. https://population.un.org/wpp/ DataQuery/. Accessed Apr 2017

6. Manobianca G, Zoccolella S, Petruzzellis A, Miccoli A, Logroscino G (2010) The incidence of major stroke subtypes in southern Italy: a population-based study. Eur J Neurol 17(9):1148-1155

7. Musolino R, La Spina P, Serra S, Postorino P, Calabró S, Savica R, Salemi G, Gallitto G (2005) First-ever stroke incidence and 30-day case fatality in the Sicilian Aeolian archipelago, Italy. Stroke 36(12):2738-2741

8. Santalucia P, Baviera M, Cortesi L, Tettamanti M, Marzona I, Nobili A, Riva E, Fortino I, Bortolotti A, Merlino L, Roncaglioni MC (2015) Epidemiologic trends in hospitalized ischemic stroke from 2002 to 2010: results from a large Italian population-based study. J Stroke Cerebrovasc Dis 24(8):1917-1923

9. Manobianca G, Zoccolella S, Petruzzellis A, Miccoli A, Logroscino $\mathrm{G}$ (2008) Low incidence of stroke in southern Italy: a populationbased study. Stroke 39:2923-2928

10. Ricci S, Celani MG, Righetti E, Cantisani TA, Bregnocchi A, Mazzoli T (2011) Incidence of stroke in the Trasimeno area: the SEPIVAC 2 study. Cerebrovasc Dis 11(Suppl. 4):5

11. Di Carlo A, Inzitari D, Galati F et al (2003) A prospective community-based study of stroke in southern Italy: the Vibo Valentia Incidence of Stroke Study (VISS). Methodology, incidence and case fatality at 28 days, 3 and 12 months. Cerebrovasc Dis 16:410-417

12. La Spina P, Savica R, Serra S et al (2008) Long-term survival and outcome after first stroke in the Sicilian Aeolian Islands archipelago population. Neurol Sci 29:153-156

13. Sacco S, Stracci F, Cerone D, Ricci S, Carolei A (2011) Epidemiology of stroke in Italy. Int J Stroke 6(3):219-227

14. Janes F, Gigli GL, D'Anna L, Cancelli I, Perelli A, Canal G, Russo V, Zanchettin B, Valente M (2013) Stroke incidence and 30-day and six-month case fatality rates in Udine, Italy: a population-based prospective study. Int J Stroke 8(Suppl A100):100-105

Publisher's note Springer Nature remains neutral with regard to jurisdictional claims in published maps and institutional affiliations. 